\title{
A new suite of tnaA mutants suggests that Escherichia coli tryptophanase is regulated by intracellular sequestration and by occlusion of its active site
}

\author{
Gang Li and Kevin D Young*
}

\begin{abstract}
Background: The Escherichia coli enzyme tryptophanase (TnaA) converts tryptophan to indole, which triggers physiological changes and regulates interactions between bacteria and their mammalian hosts. Tryptophanase production is induced by external tryptophan, but the activity of TnaA is also regulated by other, more poorly understood mechanisms. For example, the enzyme accumulates as a spherical inclusion (focus) at midcell or at one pole, but how or why this localization occurs is unknown.

Results: TnaA activity is low when the protein forms foci during mid-logarithmic growth but its activity increases as the protein becomes more diffuse, suggesting that foci may represent clusters of inactive (or less active) enzyme. To determine what protein characteristics might mediate these localization effects, we constructed 42 TnaA variants: 6 truncated forms and 36 missense mutants in which different combinations of 83 surface-exposed residues were converted to alanine. A truncated TnaA protein containing only domains D1 and D3 (D1D3) localized to the pole. Mutations affecting the D1D3-to-D1D3 interface did not affect polar localization of D1D3 but did delay assembly of wild type TnaA foci. In contrast, alterations to the D1D3-to-D2 domain interface produced diffuse localization of the D1D3 variant but did not affect the wild type protein. Altering several surface-exposed residues decreased TnaA activity, implying that tetramer assembly may depend on interactions involving these sites. Interestingly, changing any of three amino acids at the base of a loop near the catalytic pocket decreased TnaA activity and caused it to form elongated ovoid foci in vivo, indicating that the alterations affect focus formation and may regulate how frequently tryptophan reaches the active site.
\end{abstract}

Conclusions: The results suggest that TnaA activity is regulated by subcellular localization and by a loop-associated occlusion of its active site. Equally important, these new TnaA variants are immediately available to the research community and should be useful for investigating how tryptophanase is localized and assembled, how substrate accesses its active site, the functional role of acetylation, and other structural and functional questions.

Keywords: TnaA, tryptophanase, Cell pole, Protein localization, Enzyme regulation

\section{Background}

The enzyme tryptophanase hydrolyzes tryptophan to form indole, pyruvate and ammonia, and is produced by over 85 gram-positive and gram-negative bacteria [1,2]. The indole thus produced diffuses across cell membranes independent of specific transporters and affects

\footnotetext{
* Correspondence: kdyoung@uams.edu

Department of Microbiology and Immunology, University of Arkansas for Medical Sciences, Little Rock, AR 72205-7199, USA
}

the physiology of nearby members of the microbial community or their animal hosts [2-6]. For example, indole regulates such diverse microbial processes as motility, biofilm formation, antibiotic resistance, persister formation and host cell invasion [2]. Recent observations continue to underscore the biological significance of this compound, including the fact that some bacteria which do not themselves produce indole nevertheless detect its presence and become more antibiotic resistant $[7,8]$, and 
in others indole inhibits quorum sensing and growth [9]. Finally, in situ bacterial production of indole helps create and maintain proper epithelial cell function in the mammalian intestine $[10,11]$. This broad range of intra- and inter-kingdom effects makes it important to understand more fully how indole synthesis is regulated.

In Escherichia coli, tryptophanase is encoded by the tna $A$ gene in the tnaCAB operon, which also includes the tryptophan-specific transporter TnaB [12-14]. Transcription of tnaAB is activated by the cAMP-CRP complex [15], and is induced by tryptophan via a Rhodependent terminator and a $\mathrm{TnaC}$ leader peptide $[16,17]$. Induction of the tnaA gene and production of indole requires high concentrations of tryptophan, achieved mainly by the import of exogenous tryptophan via the $\mathrm{TnaB}$ transporter $[18,19]$. Functional TnaA is tetrameric, with a pyridoxal phosphate (PLP) coenzyme covalently linked to the Lys270 residue at each of the four active sites [20,21]. Incubation of the enzyme at low temperature results in reversible loss of PLP, with the tetramer disassociating into inactive dimers [22]. Two crystal structures of apo TnaA have been solved; both are tetramers in which each of the four TnaA proteins has a similar quaternary structure comprised of three subdomains: D1, D2 and D3 [23,24]. In turn, subdomains D1 and D3 form a small domain, with D2 forming a single large domain. Interactions between these two domains and with the other three TnaA subunits create the tightly assembled tetramer. The two extant TnaA crystal structures differ in that the catalytic pocket of one is closed [23] while that of the other is open [24]. The existence of these two forms suggests that the structure of TnaA is flexible, though the causes and consequences of this difference are unknown.

Recently, we found that wild type TnaA co-purifies with membrane vesicles derived from the cell poles of $E$. coli and that TnaA-sfGFP forms a single focus at midcell or at one of the poles during mid-log growth but becomes diffusely localized as the cells approach stationary phase [25]. Untagged TnaA competes with TnaA-sfGFP for polar localization, the foci do not have the characteristics of inclusion bodies, and TnaA-sfGFP is as active as the untagged protein with regard to indole production, all of which suggest that TnaA naturally localizes to the pole [25]. However, the mechanism that drives the formation of these foci is unknown, as is the question of whether they have a biological function.

Here, we show that indole production is associated with the disappearance of discrete TnaA foci and the concomitant rise of diffusely localized cytoplasmic TnaA. In tandem, we created six truncated variants of TnaA and 36 missense mutants in which different combinations of 83 surface-exposed polar amino acid residues were converted to alanine. Several variants displayed different localization characteristics and/or reduced enzymatic activities, suggesting that the sequestration and release of TnaA from intracellular foci may represent a novel posttranslational mechanism to regulate tryptophanase activity. Finally, three mutations at the base of an external loop may alter TnaA activity by regulating the frequency with which this loop occludes the enzyme's active site.

\section{Results}

\section{Diffuse TnaA protein is functional}

TnaA forms a single polar or midcell focus in all cells during mid-logarithmic growth, but the protein gradually diffuses into the cytoplasm as the culture nears stationary phase so that the majority of cells do not contain TnaA foci [25]. Interestingly, indole production is low during early exponential growth but increases dramatically as TnaA becomes more diffuse $[25,26]$. Under these same conditions, TnaA forms polar foci in $100 \%$ of the cells during exponential growth, but when these cells enter stationary phase TnaA diffuses so that only 31$58 \%$ of cells contain individual foci [25]. Thus, indole production may increase because more enzyme is made or because TnaA is moved from intracellular foci to become soluble and active in the cytoplasm. In these previous experiments, E. coli was grown in LB medium and transited rapidly from mid-logarithmic growth into stationary phase, so it was difficult to determine if the diffuse TnaA fraction was responsible for indole production. Therefore, to address this relationship, we incubated $E$. coli in M9 minimal medium to slow cell growth. We also examined TnaA function in E. coli GL619, a strain in which the chromosomal tnaA-sfgfp gene is controlled by a tufA promoter. In this strain, the TnaA-sfGFP fusion protein is produced steadily and constitutively [19], allowing us to observe the regulation of TnaA activity in the absence of any effects that might be caused by alterations in tnaA gene expression. As expected, TnaA-sfGFP formed single polar or mid-cell foci during early mid-logarithmic growth (Figure $1 \mathrm{~A}$ and B, $2 \mathrm{~h}$ ) but became completely diffuse as the cells entered stationary phase and thereafter (Figure 1B, $4 \mathrm{~h}$ and afterwards). Most interestingly, indole was not produced during mid-logarithmic growth (Figure 1C, $2 \mathrm{~h}$ ) but was produced continuously after TnaA had become diffuse in stationary phase (Figure $1 C$, $4 \mathrm{~h}$ and afterwards). These results suggested that indole was produced by the diffuse TnaA fraction but was not produced by TnaA sequestered in a focus.

TnaA probably dimerizes before assembling into the functional tetramer. Thus, the diffuse and focus fractions might be composed of different multimeric forms of TnaA, and, if so, the formation of TnaA foci might regulate TnaA function. Unfortunately, repeated attempts to isolate and analyze intact foci were unsuccessful because the foci dissolved and disappeared when the cells were disrupted. This prevented us from 


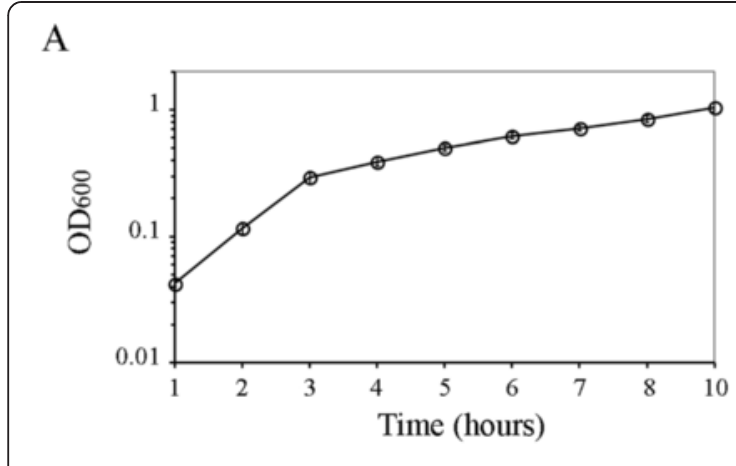

B

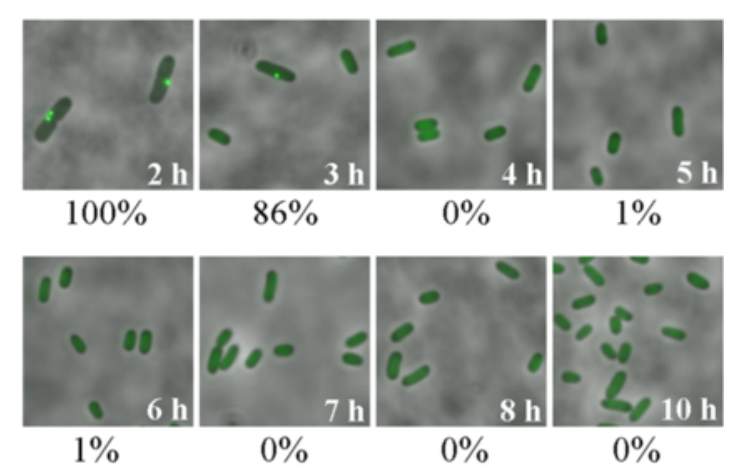

$\mathrm{C}$

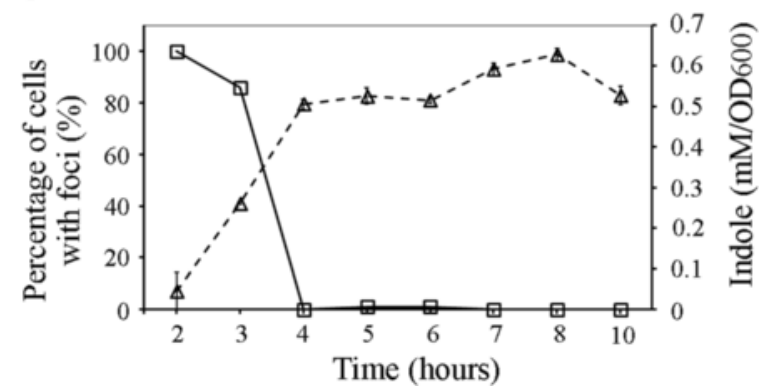

Figure 1 Indole is produced when TnaA becomes diffuse. E. coli cells (GL619) expressing TnaA-sfGFP from the constitutive tufA promoter were grown in M9 minimal medium supplemented with $1 \%$ casamino acids and $0.5 \mathrm{mM}$ tryptophan. A. Cell growth $\left(\mathrm{OD}_{600}\right)$. B. TnaA-sfGFP

localization at different times. GFP fluorescence and phase contrast images are overlaid. The percentage of the population that has a polar or midcell focus is indicated below each panel; 50-100 cells were examined for each sample. C. TnaA-sfGFP focus formation (squares) plotted against amount of indole produced (triangles). The percentage of cells with TnaA-sfGFP foci are plotted from the data in B. Three independent cultures were assayed for indole production, and the mean \pm standard deviation is presented.

analyzing the multimerization status of TnaA in foci and also prevented us from determining the precise amount of soluble TnaA in cells as they progressed through the growth cycle. However, because these lysates did convert tryptophan to indole (not shown), the results indicated that foci were easily disrupted to give fully active TnaA-sfGFP tetramers.
The TnaA D1D3 two domain dimer localizes to cell poles

Because the TnaA in discrete foci could not be tested separately from the diffuse protein, we used a truncation and mutagenesis approach to address the question of how TnaA multimerization might affect enzyme localization and activity. We rearranged the tnaA gene to create a set of derivatives that encoded all six possible combinations of the D1, D2 and D3 protein subdomains (Figure 2). The $s f g f p$ gene was appended to the 3 ' end of each rearranged gene, and the fusions were inserted into the chromosome to replace the wild type tnaA gene. The resulting gene constructs were expressed under control of the native tnaA promoter and ShineDalgarno sequence. We modeled the structures of these TnaA variants by subtracting individual subdomains from the known crystal structure (Figure 3A). The truncations predicted the existence of three potential dimers (D1-D1, D3-D3 and D1D3-D1D3) (Figure 3A, upper right three structures) and three tetramers (created by self-association of the D2, D1D2 or D2D3 domains) (Figure 3A, lower right three structures). Note that the tetramer form of TnaA required the presence of the D2 domain, but that different dimers could assemble in its absence.

Cells expressing the D1, D1D2 and D1D3 proteins produced fluorescent products (Figure 3C), but little or no fluorescent signal was observed in cells carrying the D2, D3 and D2D3 fusion products (not shown), suggesting that these latter proteins were not expressed or were degraded. SDS-PAGE analysis of the fusion proteins was

D1D2
D1D3
Figure 2 Construction of TnaA truncation mutants. The regions of
the D1, D2 and D3 subdomains were determined according to the
TnaA crystal structure (PDB 20QX). The position of the active site
Lys270 is indicated. In D2, D3 and D2D3, the starting methionine
codon was retained. In D1D3, a short linker (Ala-Ala-Gly-Tyr-Asp) was
inserted between the two subdomains. All proteins were constructed
to contain a C-terminal sfGFP. Each gene was inserted into the
chromosome to replace the wild type tnaA gene at that position.




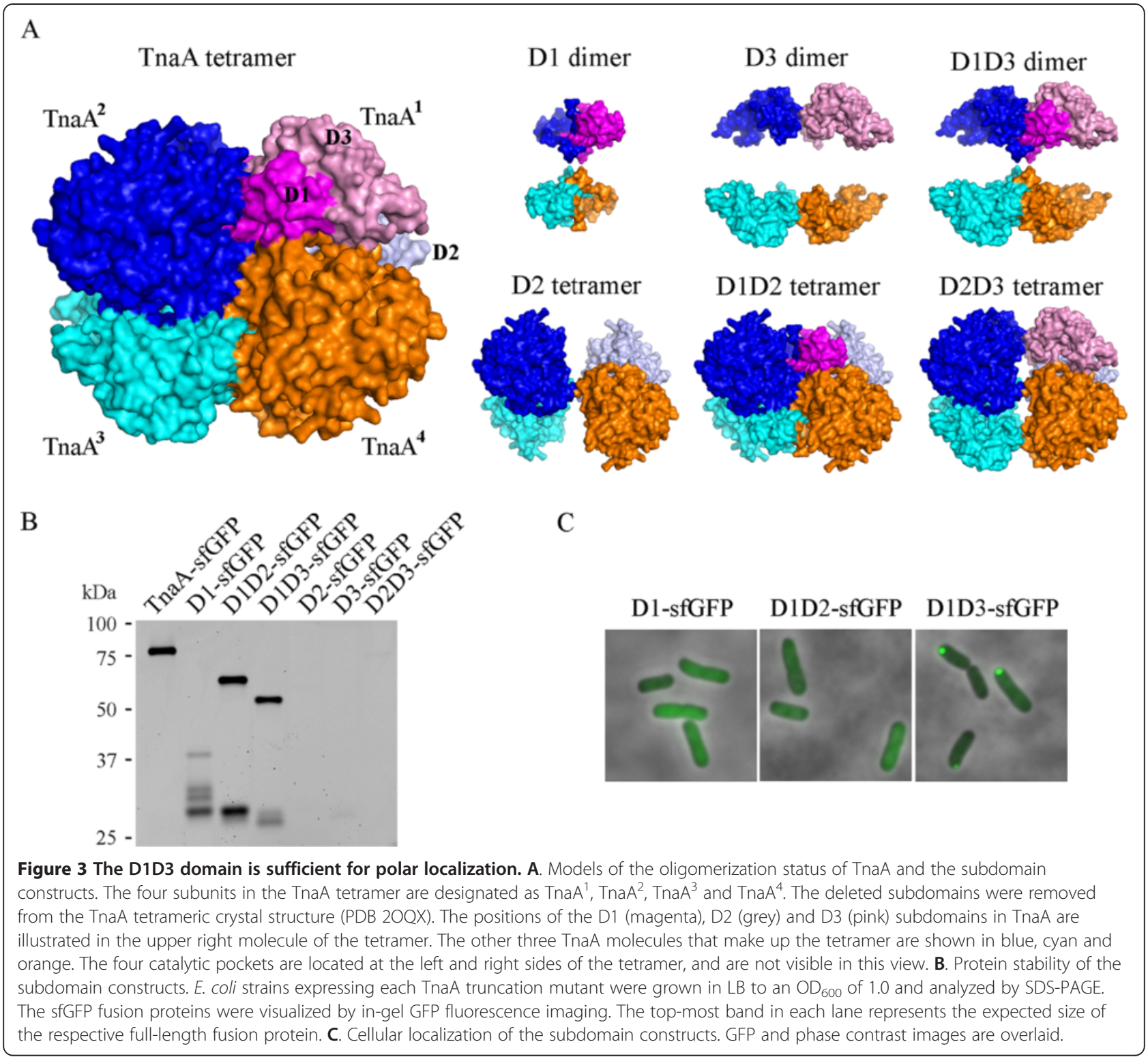

consistent with these results (Figure 3B). Among the constructs, D1D3 was the most stable (e.g., exhibited the least degradation), D1D2 was of intermediate stability, while most of the D1 domain construct was degraded into smaller fragments (Figure 3B). The D2, D3 and D2D3 subdomain variants were not visible on SDSPAGE, strongly suggesting that the D1 domain was essential for the folding or stability of TnaA. None of the truncation variants could produce indole (not shown), confirming that the complete TnaA protein was required for enzyme activity. The D1D3-sfGFP fusion protein formed a single focus at midcell or at one of the poles, as was observed for full length TnaA-sfGFP (Figure 3C). In contrast, the D1 and the D1D2 variants were diffuse throughout the cytoplasm (Figure 3C). Thus, the D1D3 dimer was sufficient for directing polar localization. Because the D1-only and D3-only variants were not stable, we could not determine directly if one or the other subdomain contained the major localization signal.

The polar localizations of TnaA and D1D3 are not artifactual TnaA-sfGFP appears to localize specifically to cell poles because the protein does not co-localize with IbpA, which binds to inclusion bodies, and because TnaAsfGFP foci are chased from the poles by untagged TnaA [25]. In addition, the dissipation of TnaA foci when the cells were broken open (see above) is unlike the behavior of inclusion bodies, which cohere and can be pelleted by 
low speed centrifugation. Nonetheless, to determine if the D1D3-sfGFP foci were inclusion bodies, the fusion protein was expressed in the presence of IbpA-mCherry, which binds to misfolded proteins and marks such artifacts $[27,28]$. Very little IbpA-mCherry signal was observed (not shown), indicating that few misfolded proteins were present to induce ibpA. In addition, when the fluorescence images were artificially enhanced, any IbpA-mCherry foci that were present did not overlap with the D1D3-sfGFP foci, confirming that the latter were not non-specific inclusion bodies (Figure 4A). In addition, we overproduced untagged wild type TnaA (from plasmid pTnaA) and found that this protein displaced polar D1D3-sfGFP foci so that the fusion protein was distributed diffusely throughout the cytoplasm (Figure 4B). These results strongly suggest that, like wild type TnaA, the localization of D1D3-sfGFP at the poles was real and specific.

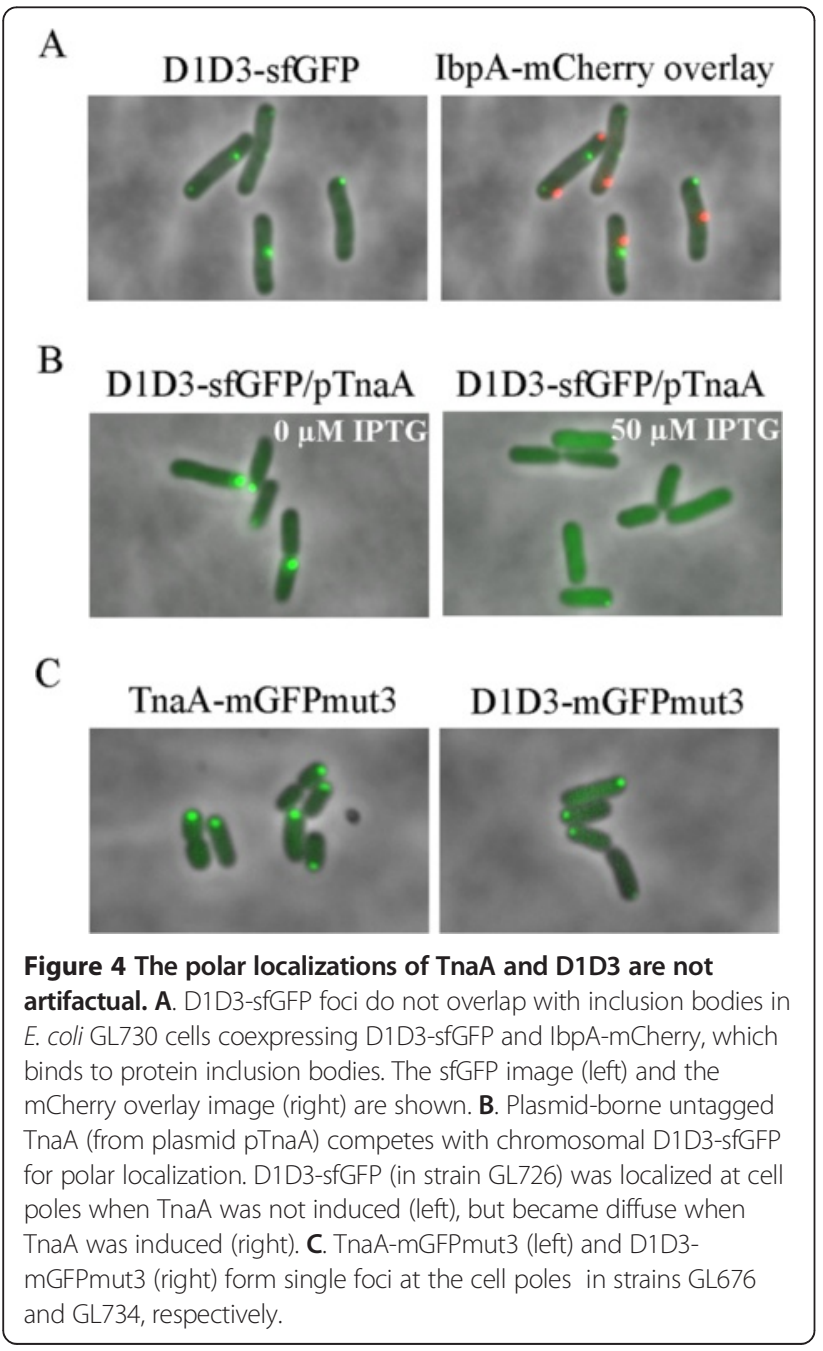

Recent observations indicate that some fluorescent reporter proteins, including sfGFP, may skew the in vivo localization behavior of proteins on a case-by-case basis [29]. For example, sfGFP-tagged ClpP forms a single focus but untagged ClpP and a ClpP-mGFPmut3 fusion protein are instead spread diffusely throughout the cytoplasm [29]. Thus, to be doubly certain that polar localization of TnaA was not an artifact caused by attaching sfGFP, we appended monomeric mGFPmut3 to the carboxyl termini of TnaA and D1D3. As before, the fusion proteins localized to the cell poles (Figure 4C), providing further evidence that the polar localization of TnaA was not an artifact caused by adding the sfGFP reporter. In subsequent localization experiments, we observed the behavior of sfGFP-fused proteins because mGFPmut3 produced a much weaker fluorescent signal.

Residues that affect assembly and localization of TnaA foci To identify the functional roles of the regions and residues of TnaA, we mutated 79 charged and polar residues on the surface of the D1D3 construct, reasoning that one or more of these residues might form ionic or hydrogen bonds with neighboring TnaA subunits or with other molecules. These residues covered almost the entire surface of the D1D3 protein (Figure 5A, residues colored yellow, blue and cyan). Non-polar residues were not mutated, although they might contribute additional interactions (Figure 5A, residues in magenta). In addition, six lysine residues (Lys5, Lys450, Lys459 on the D1D3 domains; and Lys115, Lys156, Lys239 on the D2 domain) are known to be acetylated, though the physiological functions of this modification are not known [30,31]. Therefore, in addition to the lysine residues already changed on D1D3, we also mutated the three lysine residues on the D2 domain. Finally, we mutated the active site Lys270 on the D2 domain to determine if PLP or substrate binding might affect protein localization. In sum, 83 different residues were replaced with alanine. As a preliminary screen, we constructed TnaA variants containing one to four mutations near enough to one another that they could be altered by using a single oligonucleotide during site-directed mutagenesis (Tables 1, 2 and 3, mutants A1-TnaA to A32TnaA). A corresponding series of mutagenized variants was engineered into the D1D3 fusion protein (Tables 1, 2 and 3, A1-D1D3 to A32-D1D3). sfGFP was fused to the carboxyl terminus of each TnaA or D1D3 variant, and the gene for each mutant was inserted into the original chromosomal position so that gene expression was controlled by the native tnaA promoter.

The localization and enzymatic phenotypes of the mutant proteins fell into three categories. In the first category, the mutant proteins behaved like wild type TnaA (Table 1). Each of these variants formed a single polar or 


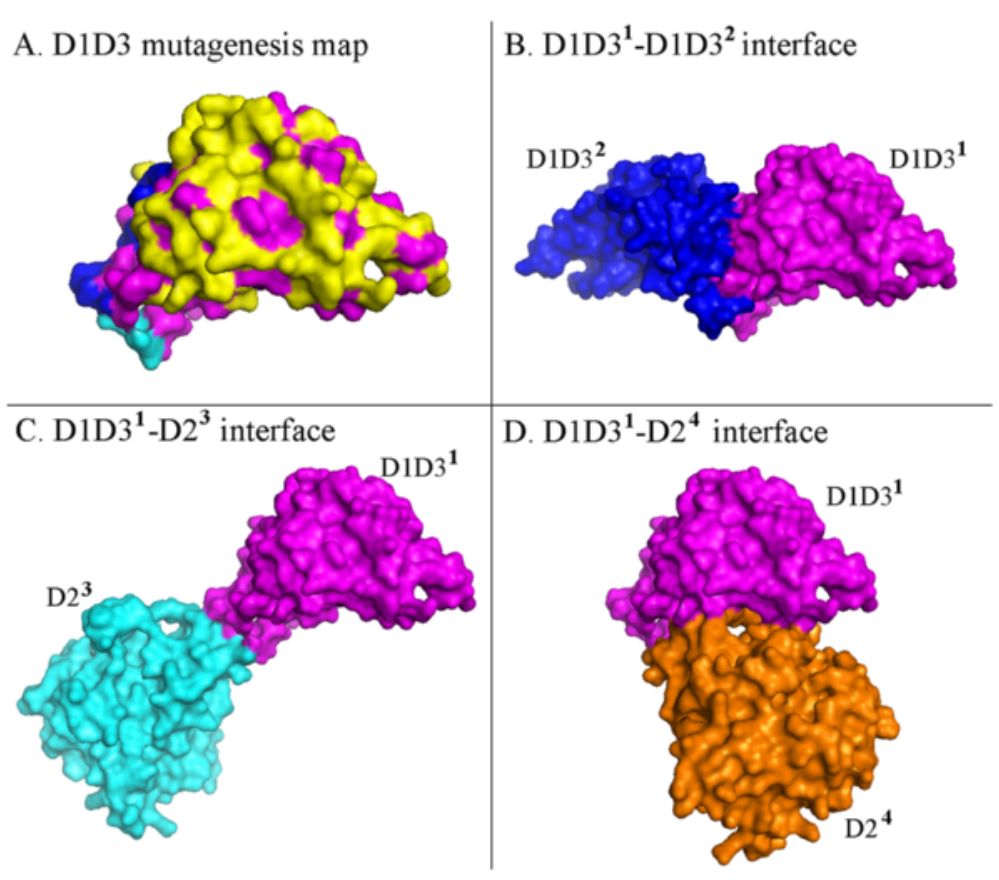

Figure 5 D1D3 surface residues that affect polar localization of TnaA-sfGFP. A. Magenta: residues that were not mutagenized. Yellow: alanine substitution mutations that did not affect polar localization of D1D3 or TnaA. Deep blue: mutations that delayed assembly of TnaA foci (mutation sets A2-TnaA, A24-TnaA and A25-TnaA). Cyan: mutations that produced diffuse localization of the D1D3-sfGFP TnaA construct (A9-D1D3). Mutations that produced ovoid foci are not visible in this view and are illustrated in Figure 7. The interfaces between D1D3 ${ }^{1}$ and other subunits are illustrated in $\mathbf{B}$ to D. Note that mutations causing delayed focus disassembly at stationary phase (Table 3) are not illustrated.

midcell focus during the mid-to-late growth phase, after which the proteins became diffuse as the cells entered stationary phase. In addition, these proteins produced indole at wild type levels, indicating that they had normal enzymatic activity. Of particular note is that neither localization nor enzyme activity was affected by mutating individual lysine residues that would normally be acetylated (Table 1, mutants A1, A10, A11, A12, A28 and A30). In fact, a mutant in which all six acetylated lysine residues were mutated continued to exhibit wild type localization and its enzymatic activity was affected very little (Table 1, mutant A33). Thus, many surface residues did not contribute significantly to TnaA function, and the purpose of lysine acetylation remains unknown.

The second mutant category consisted of TnaA variants that exhibited altered localization and/or enzymatic properties in cells as they reached the mid-to-late growth phase (Table 2). These mutants could be classified into three subgroups. The first subgroup consisted of full length proteins that exhibited delayed focus formation (Table 2, variants A2, A24 and A25). These variants were distributed diffusely in the cytoplasm during mid-log growth and formed a single polar or midcell focus about 30 min later than did wild type TnaA (e.g.,
Figure 6A). The mutated residues in these variants were located along the interface of neighboring D1D3-toD1D3 domains (Figure 5A, deep blue residues), suggesting that interactions along the non-catalytic axis of the TnaA tetramer [32] might be affecting in vivo protein sequestration. The second subgroup, represented by only one mutant, localized normally when incorporated into the full length TnaA protein (Table 2, mutant A9, and Figure 6C). However, the corresponding A9-D1D3 truncation variant produced no foci but was, instead, distributed throughout the cytoplasm at all times (Table 2, and Figure 6D). The mutations in this variant were clustered tightly at the $\mathrm{D} 1 \mathrm{D} 3^{1}-\mathrm{D} 2^{3}$ interface (Figure $5 \mathrm{~A}$, residues in cyan). However, the D2 domain was not present in this construct so that these residues were exposed on the surface of the A9-D1D3 truncation protein. Thus, these residues might be required for formation of dimers and foci. Finally, members of the third subgroup consisted of TnaA variants that produced highly elongated ovoid foci in the context of the full length mutant protein (Table 2, variants A21, A21a, A23, A23a, A31 and A31a). These ovoid foci (e.g., Figures $6 \mathrm{E}$ and $7 \mathrm{~A}$ ) were much different from the spherical foci associated with wild type TnaA and other variants (e.g., Figure $6 \mathrm{C}$ and F). These variants formed a single focus at midcell or at 
Table 1 Mutations that do not affect TnaA localization or enzyme activity (Category 1)

\begin{tabular}{|c|c|c|c|c|c|}
\hline Variant & Residue(s) changed to alanine & Residue location $^{a}$ & TnaA-sfGFP localization $^{\text {b }}$ & D1D3-sfGFP Localization ${ }^{c}$ & Indole $^{d}(\%)$ \\
\hline$\overline{\mathrm{A} 1}$ & $\mathrm{~K} 5$ & D1 surface & Wild type & $N / A^{e}$ & 96.5 \\
\hline A3 & E17, K20, R21 & D1 surface & Wild type & Wild type & 101.3 \\
\hline A4 & T23, R24, Y26 & D1 surface & Wild type & Wild type & 99.5 \\
\hline A6 & K33, S34 & D1 surface & Wild type & Wild type & 102.7 \\
\hline A10 & K115 & D2 surface & Wild type & N/A & 93.0 \\
\hline A11 & K156 & D2 surface & Wild type & N/A & 98.7 \\
\hline A12 & K239 & D2 surface & Wild type & N/A & 100.1 \\
\hline A14 & N327, D329 & D3 surface & Wild type & Wild type & 96.8 \\
\hline A15 & Q339, Y340, D343 & D3 surface & Wild type & Wild type & 99.6 \\
\hline A16 & E346, E347 & D3 surface & Wild type & Wild type & 93.1 \\
\hline A18 & D363, K366 & D3 surface & Wild type & Wild type & 97.7 \\
\hline A19 & H370, D374, Q375 & D3 surface & Wild type & Wild type & 99.2 \\
\hline A21b & R403 & D3 loop & Wild type & N/A & 103.3 \\
\hline A21C & D404 & D3 loop & Wild type & N/A & 105.4 \\
\hline$A 22$ & K406, K409, Q410 & D3 loop & Wild type & Wild type & 100.9 \\
\hline A26 & E437, K440, H441 & D3 surface & Wild type & Wild type & 92.7 \\
\hline A27 & K443, E444, N445, N448 & D3 surface & Wild type & Wild type & 103.4 \\
\hline A28 & K450 & D3 surface & Wild type & N/A & 97.9 \\
\hline A30 & K459 & D3 surface & Wild type & N/A & 102.9 \\
\hline$A 31 C$ & T465 & D3 surface & Wild type & N/A & 72.7 \\
\hline A32 & K467, K469, E470 & D3 surface & Wild type & Wild type & 92.4 \\
\hline A33 & K5, K115, K156, K239, K450, K459 & Surface & Wild type & Wild type & 82.5 \\
\hline
\end{tabular}

Residue location is based on the crystal structure of TnaA (PDB ID 2OQX). Pocket, the active site cavity; loop, the loop comprised of aa 398 to 416 at the edge of the catalytic pocket.

${ }^{b}$ Wild type: spherical focus at midcell or one of the poles at mid-to-late exponential phase, becoming more diffuse at stationary phase as wild type TnaA.

'Wild type: spherical focus at midcell or one of the poles at mid-to-late exponential phase. D1D3 constructs were mostly degraded at stationary phase.

${ }^{\mathrm{d}}$ The amount of indole produced by each TnaA-sfGFP variant compared to that produced by wild type TnaA in LB at an OD 600 of 1.5 . Values are reported as the percentage of wild type, and are the average of two independent cultures. Growth rates of all cells carrying wild type TnaA or any of its variants were nearly identical.

${ }^{\mathrm{e}} \mathrm{N} / \mathrm{A}$ indicates that the variant was not constructed or that data was not available.

Table 2 Mutations that alter TnaA focus formation during exponential growth (Category 2)

\begin{tabular}{|c|c|c|c|c|c|}
\hline Variant & Residue(s) changed to alanine & Residue location $^{a}$ & TnaA-sfGFP localization $^{\text {b }}$ & D1D3-sfGFP Localization ${ }^{c}$ & Indole $^{d}(\%)$ \\
\hline$A 2$ & $\mathrm{E} 9, \mathrm{R} 12, \mathrm{R} 14$ & D1D3 ${ }^{1}-$ D1D3 $^{2}$ interface & Delayed & Wild type & 7.2 \\
\hline A24 & T426, Y427, T428 & D1D3 ${ }^{1}-D 1 D 3^{2}$ interface & Delayed & Wild type & 8.5 \\
\hline A25 & Q429, T430, H431, D433 & D1D3 ${ }^{1}-D 1 D 3^{2}$ interface & Delayed & Wild type & 13.3 \\
\hline A9 & T60, Q61, S62, Q64 & $D 1 D 3^{1}-D 2^{3}$ & Wild type & Diffuse & 5.3 \\
\hline A21 & S398, R403, D404 & Edge of the pocket & Ovoid & Wild type & 5.8 \\
\hline A21a & S398 & Bottom of the loop & Ovoid & Wild type & 43.4 \\
\hline A23 & E416, R419 & Edge of the pocket & Ovoid & Wild type & 5.1 \\
\hline A23a & E416 & Bottom of the loop & Ovoid & $N / A^{e}$ & 12.1 \\
\hline A31 & R462, H463, T465 & Edge of the pocket & Ovoid & Wild type & 2.1 \\
\hline A31a & R462 & Bottom of the loop & Ovoid & N/A & 57.1 \\
\hline
\end{tabular}

Legend is the same as for Table 1. 
Table 3 Mutations that delay TnaA focus disassembly in stationary phase (Category 3)

\begin{tabular}{|c|c|c|c|c|c|}
\hline Variant & Residue(s) changed to alanine & Residue location $^{\mathrm{a}}$ & TnaA-sfGFP localization $^{\text {b }}$ & D1D3-sfGFP localization ${ }^{c}$ & Indole $^{d}(\%)$ \\
\hline$\overline{\mathrm{A} 2}$ & E9, R12, R14 & D1D3 ${ }^{1}$-D1D3 ${ }^{2}$ interface & Delayed & Wild type & 7.2 \\
\hline A5 & R27, E28, E29 & D1 surface & Wild type & Wild type & 14.9 \\
\hline A7 & D42, S43, E44, D45 & D1 surface & Wild type & Wild type & 36.8 \\
\hline A8 & D49, T52, D53, S54 & D1D3 ${ }^{1}-D 2^{4}$ & Wild type & Wild type & 6.6 \\
\hline A9 & T60, Q61, S62, Q64 & $D 1 D 3^{1}-D 2^{3}$ & Wild type & Diffuse & 5.3 \\
\hline A13 & K270 & D2 active site & Wild type & $\mathrm{N} / \mathrm{A}$ & 10.9 \\
\hline A17 & C352, Q353, Q354 & D3 pocket & Wild type & Wild type & 6.0 \\
\hline \multirow[t]{2}{*}{ A20 } & E384, K387, R392 & D3 surface (EK) & Wild type & Wild type & 4.8 \\
\hline & & $\mathrm{D}^{1 D} 3^{1}-\mathrm{D} 2^{4}(\mathrm{R})$ & & & \\
\hline A21 & S398, R403, D404 & Edge of the pocket & Ovoid & Wild type & 5.8 \\
\hline A21a & S398 & Bottom of the loop & Ovoid & Wild type & 43.4 \\
\hline A29 & T453, T455, Y456, E457 & D3 surface & Wild type & Wild type & 11.7 \\
\hline
\end{tabular}

Legend is the same as for Table 1.

one pole, but the assemblies were elongated and oriented parallel to the long axis of the cell or, in some cases, were slightly tilted relative to the poles (Figures $6 \mathrm{E}$ and 7A). The mutations in these variant proteins clustered near the edge of the catalytic pocket, though they were not themselves part of the active site. A surprising observation was that some full-length TnaA variants localized differently than did their corresponding D1D3 truncation proteins (Table 2). For example, unlike their full length counterparts, focus formation was not

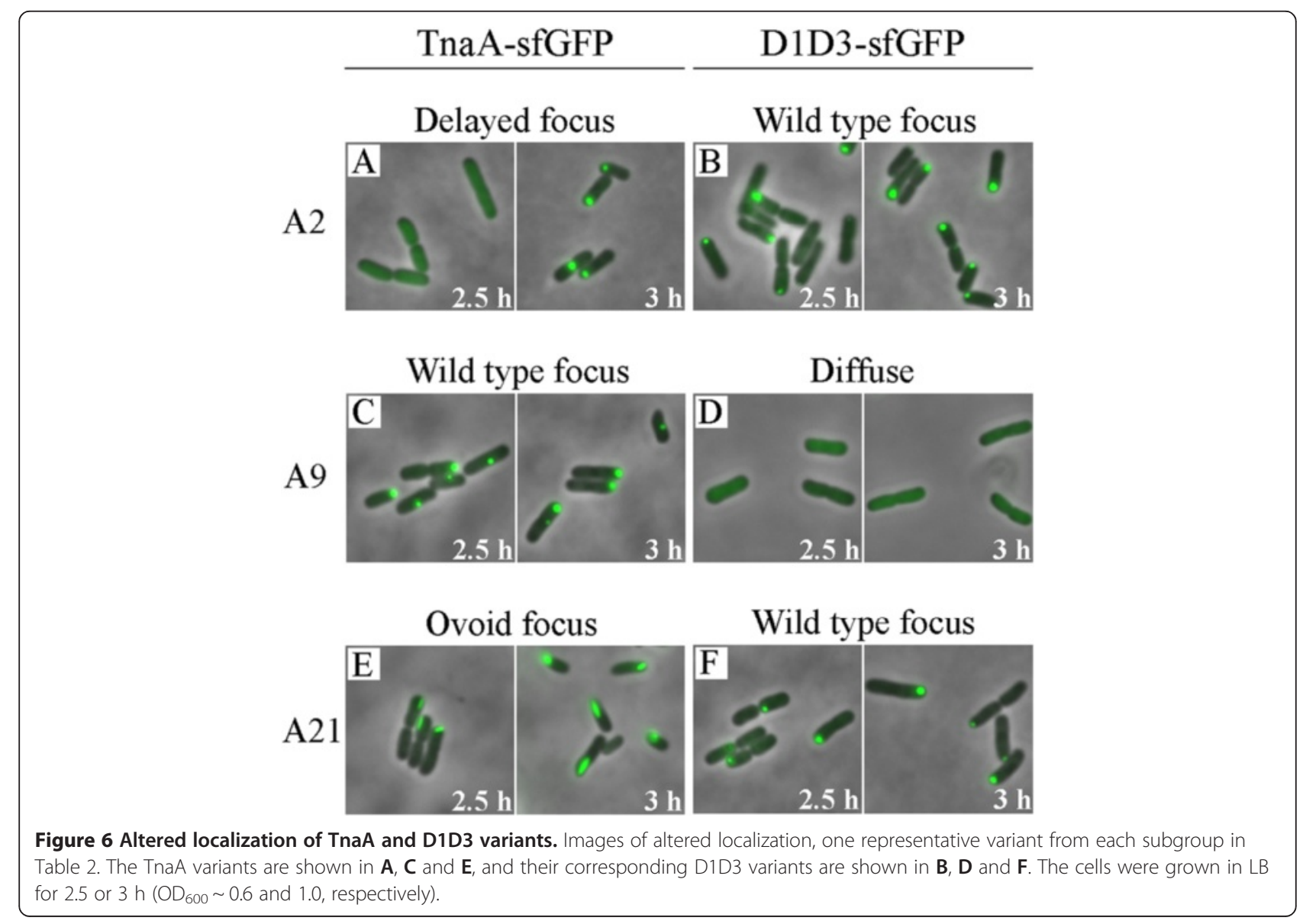




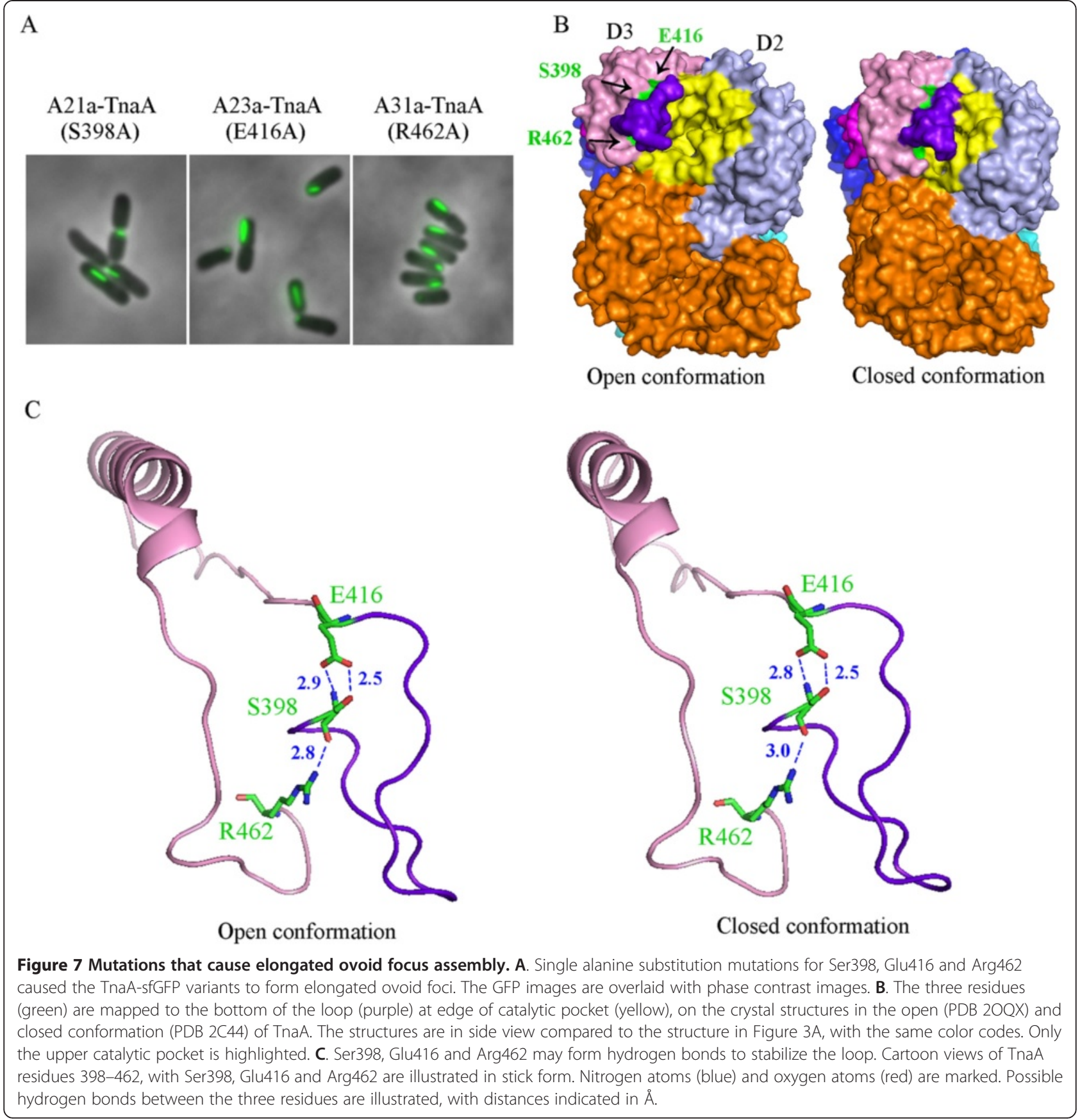

delayed for the A2-D1D3, A24-D1D3 and A25-D1D3 truncation variants (Table 2 and Figure 6B). Also, as stated above, mutations at the D1D $3^{1}-\mathrm{D} 2^{3}$ interface produced a diffuse localization of A9-D1D3 (Figure 6D) but the A9-TnaA full-length variant continued to localize normally at the poles (Figure 6C). Finally, the mutations that caused full length TnaA-sfGFP to form elongated ovoid foci had no effect on the shape of foci produced by the truncation proteins A21-D1D3, A23-
D1D3 or A31-D1D3 (Table 2, Figure 6F). These results suggest that polar localization is a complicated process with multiple interactions involving both the D1D3 and D2 domains.

The third major mutant category was composed of TnaA variants for which the disassembly of foci was delayed during stationary phase (Table 3). Previously, we showed that when wild type TnaA is expressed from its native promoter, the protein becomes more diffuse when 
entering stationary phase [25], an observation that was confirmed here because TnaA foci begin to disappear in early stationary phase cells (e.g., after 4-6 h, Table 4). In contrast, this third set of mutant proteins continued to form polar foci in almost 100\% of cells at $6 \mathrm{~h}$ and began to become diffuse only at $12 \mathrm{~h}$ (Tables 3 and 4). Three of these variants also displayed altered focus formation (Table 2), suggesting that in some cases common factors may affect both assembly and disassembly of foci. In addition, A13-TnaA, which carries a Lys270Ala substitution at the active site, localized at poles even though it had no enzymatic activity (i.e., indole was produced at background levels) (Table 3, mutant A13). Other mutations in the catalytic pocket also eliminated indole production but had no effect on polar localization (e.g., A17-TnaA, Table 3). These results indicated that localization did not depend on the presence of the PLP cofactor or on the ability of TnaA to hydrolyze tryptophan. However, altering some active site residues did affect the disassembly of foci during stationary phase.

We particularly wished to know if delayed disassembly of TnaA foci was associated with a delay in the development of enzymatic activity, as measured by the production of indole. Therefore, we examined four of these variants (A2, A9, A17 and A21a) for enzymatic activity and for the presence of TnaA-sfGFP foci (Table 4). Each of the variants described in Tables 3 and 4 produced less indole at all points in the growth cycle, even though the amount of each variant protein was 2- to 3-fold higher per cell after $12 \mathrm{~h}$ incubation (not shown). In terms of TnaA localization, the selected proteins exhibited three types of behavior. First, the A21a variant most closely replicated the cycle of wild type TnaA-sfGFP. Although A21a foci disappeared later than did wild type TnaA foci, after $12 \mathrm{~h}$ the number of foci and enzymatic activity paralleled the results observed for wild type TnaA (Table 4, variant A21a). The A21a variant carries the S398A mutation, which is unable to close the peptide loop adjacent to the active site (Figure 7C). This implies that although the loop may inhibit substrate access to

Table 4 Indole activity of selected disassembly-delay mutants of TnaA

\begin{tabular}{|c|c|c|c|c|c|c|c|c|c|c|}
\hline \multirow[t]{2}{*}{ Variant $^{\mathrm{a}}$} & \multicolumn{5}{|c|}{ Cells with foci (\%) } & \multicolumn{5}{|c|}{ Indole production (mM) } \\
\hline & $2 \mathrm{~h}$ & $3 \mathrm{~h}$ & $4 \mathrm{~h}$ & $6 \mathrm{~h}$ & & $2 \mathrm{~h}$ & $3 \mathrm{~h}$ & $4 \mathrm{~h}$ & $6 \mathrm{~h}$ & $12 \mathrm{~h}$ \\
\hline$\pi$ & 100 & 100 & 100 & 75 & 67 & 0.02 & 0.25 & 0.55 & 0.59 & 0.50 \\
\hline A2 & 41 & 100 & 100 & 100 & 64 & 0.00 & 0.04 & 0.04 & 0.13 & 0.05 \\
\hline A9 & 100 & 100 & 100 & 100 & 72 & 0.01 & 0.05 & 0.03 & 0.12 & 0.07 \\
\hline A17 & 100 & 100 & 100 & 100 & 100 & 0.02 & 0.06 & 0.03 & 0.16 & 0.15 \\
\hline $21 \mathrm{a}$ & 100 & 100 & 100 & 100 & 87 & 0.01 & 0.06 & 0.24 & 0.43 & 0.45 \\
\hline
\end{tabular}

${ }^{a}$ The number of foci and enzyme activity of specified mutants of TnaA-sfGFP fusion proteins. the active site (see Discussion), it may not be required for enzymatic activity per se. The A2 and A9 variants displayed a second type of behavior. For each of these proteins the number of foci decreased at $12 \mathrm{~h}$, similar to wild type TnaA, but the enzymatic activity of the mutants remained low (Table 4). Therefore, these mutations may affect both localization and enzymatic activity. Finally, the A17 variant exhibited a third class of behavior - the number of cells with intact foci remained at almost $100 \%$, even at $12 \mathrm{~h}$, while enzymatic activity also remained low. This phenotype was consistent with the possibility that sequestered TnaA is inactive (see Discussion), though more work would have to be done to prove that this protein was active outside of foci.

\section{Three loop-associated substitutions produce elongated ovoid foci}

We were very intrigued by the formation of elongated ovoid foci in the A21-TnaA, A23-TnaA and A31-TnaA variants. Each of these proteins contained two to four amino acid substitutions, so we constructed single substitution variants to determine the residues responsible for this curious phenomenon. Each of three alanine substitutions replacing Ser398 (A21a-TnaA), Glu416 (A23a-TnaA) or Arg462 (A31a-TnaA) resulted in protein variants that formed elongated ovoid foci and decreased indole production by $43-78 \%$ (Table 2, Figure 7A). These altered localizations and enzyme activities were not due to decreased protein stability (Figure 8).

The three relevant residues mapped to the bottom of a loop, encompassing residues 398-416, located just to the side of the TnaA catalytic pocket (Figure 7B). This loop obscures the catalytic pocket in the closed conformation of TnaA (Figure 7B, right), but is canted away from the active site cavity in the open conformation of the protein (Figure 7B, left), making it likely that movement of this loop shifts the enzyme between its open-and-closed forms. The three residues are in close contact, possibly forming hydrogen bonds to maintain the position of this loop (Figure 7C). Surprisingly, the local arrangement of these residues and the loop itself in the closed form of TnaA are almost identical to the arrangements in the open form, suggesting that the movement of the loop is mediated mainly by moving the D1D3 domain towards the D2 domain. Mutations of the three residues may alter the position or orientation of the loop or may disrupt how the loop is tethered to the body of TnaA, thereby altering the protein's enzymatic activity and localization. Interestingly, mutations that changed the composition of the loop itself (e.g., in variants A21b-TnaA, A21c-TnaA and A22-TnaA) did not affect polar localization or enzymatic activity (Table 1), suggesting that protein function 


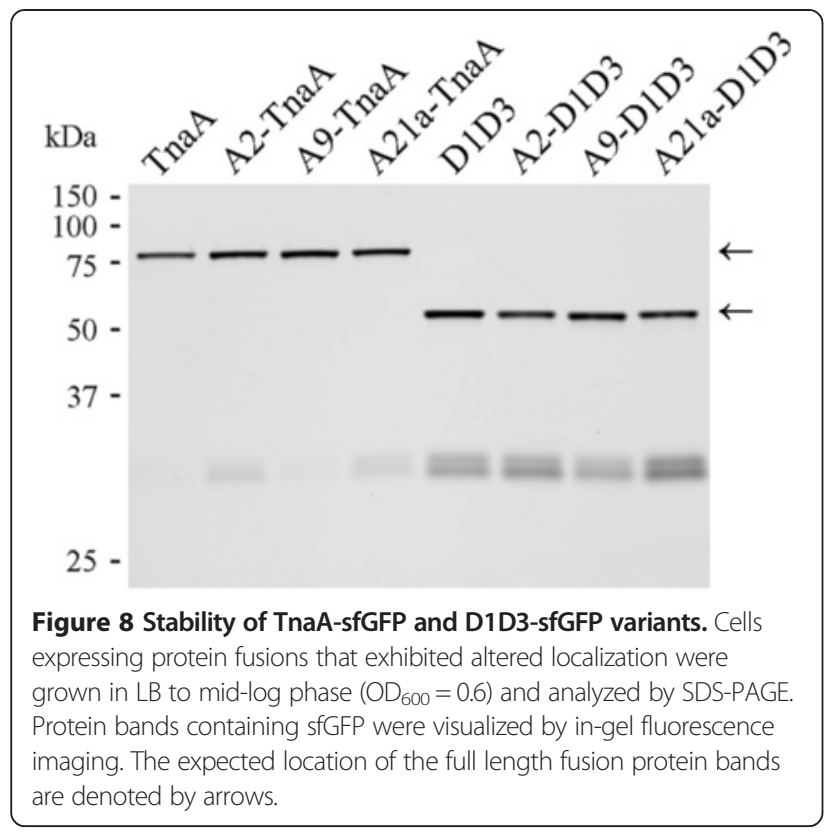

depends more on loop placement than on loop composition. The results imply that the mutated residues at the base of the loop may alter the equilibrium between the open and closed conformations of TnaA by affecting loop dynamics.

\section{Discussion}

A noteworthy number of proteins are distributed asymmetrically in bacterial cells, and these different locations may affect protein activity or function [33,34]. Such relationships are most obvious for proteins within macromolecular complexes, such as those that comprise the divisome, flagella, secretion systems and the chromosomal segregation apparatus [35-42]. It is less clear how or why other proteins are directed to specific locations, or whether differential localization affects protein function. However, a few examples lend credence to the idea that cellular localization causes or is at least associated with alterations in enzymatic activity. For example, the E. coli MurG protein participates in synthesizing peptidoglycan precursors for insertion into the cell wall, and excess amounts of this protein are localized to the cell poles, where the enzyme is apparently inactive [43]. Similarly, the activity of the Bacillus subtilis LicT antiterminator is accompanied by an overt change in its cellular localization: inactive enzyme (in the absence of the substrate salicin) is distributed diffusely in the cytoplasm, whereas active enzyme (in the presence of salicin) moves to a subpolar localization [44]. Finally, the Caulobacter crescentus CtpS protein, a CTP synthase, performs its enzymatic role when diffuse in the cytoplasm but also helps determine cell shape by polymerizing into a cytoskeletal filament along the cell membrane, this latter function being independent of its enzymatic activity [45]. We now report that the cellular location and activity of the TnaA tryptophanase are correlated, suggesting that the sequestration of TnaA in discrete foci may regulate its enzyme activity.

A strong indication that TnaA localization influences its enzymatic activity is that $E$. coli produces very little indole when TnaA is located in foci but that it produces large amounts of indole when the foci disappear and TnaA becomes diffuse throughout the cytoplasm. This suggests that the fraction of TnaA in foci is inactive and that the diffuse population is functional. A second argument supporting a relationship between protein function and focus formation is the existence of three mutations that reduce enzyme activity and change the geometry of TnaA foci from spherical to elongated ovals. The mechanism that creates these effects is unknown but the three altered residues are located at the base of a loop near the catalytic cavity, indicating that the disposition of this loop affects both characteristics. A third argument in support of the focus-function premise is that several mutations (i.e., in the mutants A2, A24 and A25) alter the kinetics or timing of focus formation and also reduce enzyme activity. The location of these mutated residues at the D1D3-to-D1D3 dimer interface, well away from the catalytic pocket, puts them in a position to affect the equilibrium among the different multimeric forms of TnaA, which may affect both sequestration and activity of the enzyme. Finally, several mutants delay the time when TnaA moves from foci to become diffuse while also delaying the time at which the enzyme becomes active. This strong genetic correlation suggests the two characteristics are causally related and corroborates the relationships observed for the wild type protein.

Overall, the results are consistent with a model in which individual foci contain inactive TnaA monomers or dimers while the diffuse fraction contains active tetramers (Figure 9). If true, then E. coli may regulate TnaA activity by altering the equilibrium between these protein states. This model is supported by previous work in which the TnaA C298S mutant, which is near the active site, disassociates more readily into inactive dimers, thus reinforcing the relationship between activity and tetramerization [32]. The fact that focus assembly is reversible, both in vivo during growth and in vitro when cells are disrupted, suggests that one or more cellular factors might help orchestrate the focus-to-diffuse shift. The physiological purpose of this regulation might be to restrict indole signaling to a particular time late in the growth cycle.

One of the most intriguing findings was that three individual missense mutations decrease the tryptophanase 


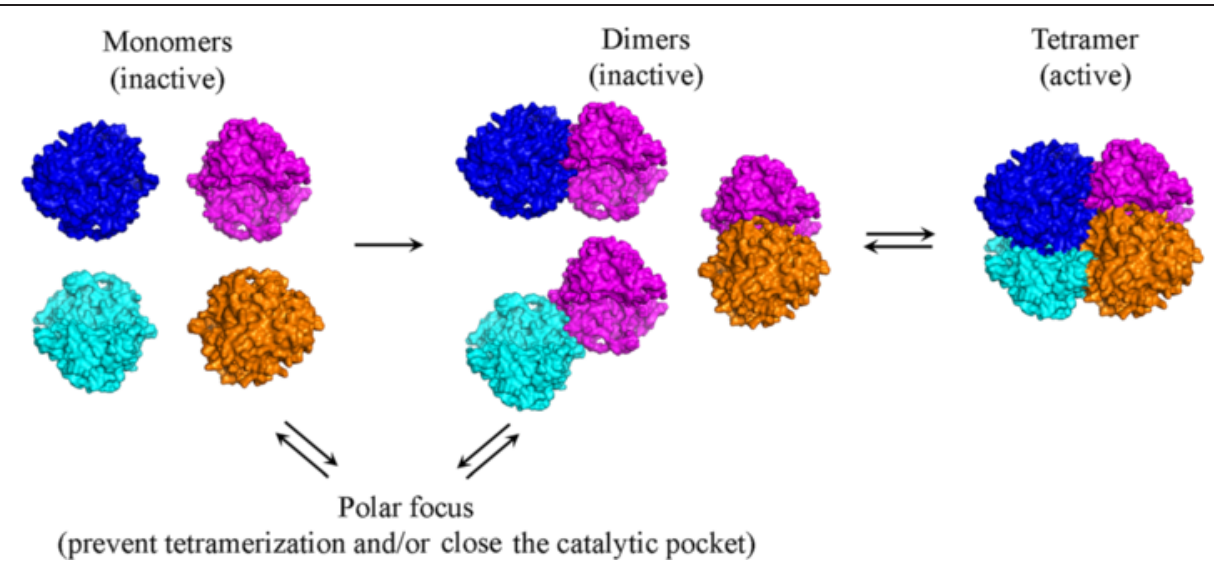

Figure 9 Model of post-translational regulation of TnaA activity by focus formation. Four TnaA units (magenta, blue, cyan and orange) were modeled as dimers and as the tetramer by using the PISA software, based on the crystal structure PDB 2OQX. Focus assembly of TnaA monomers and dimers would prevent the protein from assembling into active tetramers and/or trap the catalytic pocket in a closed conformation, thus regulating TnaA activity.

activity of TnaA and are associated with the formation of curiously elongated ovoid foci. The affected amino acids (Ser398, Glu416 and Arg462) are located at the base of a loop (from residues 398-416) that sits to one side of the catalytic pocket. In the two crystal structures of TnaA, this catalytic site is closed in one conformation and open in the other. The major cause of the closed pocket is a movement of the small domain (D1D3) towards the large domain (D2), re-orientating the loop so that it controls access to the active site by physically occluding the catalytic cavity. The three mutations that do change the behavior of TnaA foci are perfectly positioned to affect the orientation of the loop and may regulate the equilibrium between the open and closed versions of TnaA. Such alterations may also favor intermolecular interactions that lead to the formation of oddly shaped foci. Alternately, the assembly of TnaA into foci may drive the loop to adopt one or the other orientation, which could determine whether the active site is more or less available to its substrate. Mutations affecting residues within the loop itself did not alter polar localization or enzymatic activity, implying that the loop's orientation is the more important regulatory feature. However, combining any one of the three mutations at the base of the loop with mutations within the loop produced enzymes with even lower activity, suggesting that once the base is altered additional considerations come into play (Table 2, compare variants A21a, A23a and A31a to variants A21, A23 and A31). The tryptophanase of Proteus vulgaris and the tyrosine phenollyases of Citrobacter freundii and Erwinia herbicola are $51.7 \%, 41.1 \%$ and $43 \%$ identical to E. coli TnaA, respectively (EMBOSS Needle software analysis, via http://www. ebi.ac.uk/Tools/psa/emboss_needle/index.html), and all three have crystal structures with quaternary structures similar to that of E. coli TnaA (PDB entries 1AX4, 2EZ1 and 1 C7G). Significantly, in all three enzymes the residues homologous to Ser398, Glu416 and Arg462 in E. coli TnaA are conserved and map to the base of a protruding loop near the active site, suggesting that the loop might play a similar structural and regulatory role in these enzymes, as well.

Lastly, we again address the question of whether polar assembly is an artifact caused by tagging TnaA with the fluorescent protein sfGFP. Mis-localization of analogous constructs made with the Clp protease [29] and MreB [46] does not mean that all protein fusion localizations are artifactual. The lesson is that such results need to be interpreted with caution when this kind of tagging is the sole approach for studying protein localization. In the case of TnaA, additional evidence suggests that polar assembly of TnaA-sfGFP is specific and likely represents its native localization [25]. Such evidence includes the fact that untagged TnaA associates with membrane vesicles derived from poles, that it competes with TnaA-sfGFP for polar localization, and that TnaA-sfGFP foci do not overlap with inclusion bodies [25]. Here, we find that the TnaA-sfGFP foci disappear when cells are lysed, again indicating that these are not inclusion bodies. Finally, when we fused TnaA to monomeric GFP (mGFPmut3) as recommended by Landgraf et al. [29], the composite protein formed the same type of polar foci, providing additional evidence that localization is not due to artifacts caused by sfGFP.

\section{Conclusions}

The subcellular location and enzymatic activity of the TnaA tryptophanase are correlated, strongly suggesting 
Table 5 E. coli strains

\begin{tabular}{|c|c|c|}
\hline Strain & Description $^{\mathrm{a}}$ & Source or Reference \\
\hline MG1655 & Wild type $F^{-} \lambda^{-}$ilvG $r f b-50$ rph-1 & Laboratory collection \\
\hline GL38 & MG1655 aer-sfgfp::kan & [25] \\
\hline GL40 & MG1655 tnaA-sfgfp::kan & [25] \\
\hline GL60 & MG1655 ibpA-mCherry::kan & [25] \\
\hline GL504 & MG1655 $\Delta$ tnaA::frt & [25] \\
\hline GL619 & GL607 $\triangle$ tnaAB::frt $\mathrm{P}_{\text {tufA::tnaA-sfgfp thaB::frt }}$ & [19] \\
\hline GL676 & MG1655 tnaA-mgfpmut3::kan & This study \\
\hline GL678 & MG1655 D1-sfgfp::kan & This study \\
\hline GL680 & MG1655 D1D2-sfgfp::kan & This study \\
\hline GL682 & GL504 D3-sfgfp::kan & This study \\
\hline GL684 & GL504 D2D3-sfgfp::kan & This study \\
\hline GL686 & GL504 D1D3-sfgfp::kan & This study \\
\hline GL688 & GL504 D2-sfgfp::kan & This study \\
\hline GL726 & GL686 D1D3-sfgfp::frt & This study \\
\hline GL730 & GL726 D1D3-sfgfp::frt ibpA-mCherry::kan & This study \\
\hline GL734 & GL504 D1D3-mgfpmut3:.kan & This study \\
\hline GL801 & GL504 A1-tnaA-sfgfp::kan (K5A) & This study \\
\hline GL802 & GL504 A2-tnaA-sfgfp::kan (E9A, R12A, R14A) & This study \\
\hline GL803 & GL504 A3-tnaA-sfgfp::kan (E17A, K20A, R21A) & This study \\
\hline GL804 & GL504 A4-tnaA-sfgfp::kan (T23A, R24A, Y26A) & This study \\
\hline GL805 & GL504 A5-tnaA-sfgfp::kan (R27A, E28A, E29A) & This study \\
\hline GL806 & GL504 A6-tnaA-sfgfp::kan (K33A, S34A) & This study \\
\hline GL807 & GL504 A7-tnaA-sfgfp::kan (D42A, S43A, E44A, D45A) & This study \\
\hline GL808 & GL504 A8-tnaA-sfgfp::kan (D49A, T52A, D53A, S54A) & This study \\
\hline GL809 & GL504 A9-tnaA-sfgfp::kan (T60A, Q61A, S62A, Q64A) & This study \\
\hline GL810 & GL504 A10-tnaA-sfgfp::kan (K115A) & This study \\
\hline GL811 & GL504 A11-tnaA-sfgfp::kan (K156A) & This study \\
\hline GL812 & GL504 A12-tnaA-sfgfp::kan (K239A) & This study \\
\hline GL813 & GL504 A13-tnaA-sfgfp::kan (K270A) & This study \\
\hline GL814 & GL504 A14-tnaA-sfgfp::kan (N327A, D329A) & This study \\
\hline GL815 & GL504 A15-tnaA-sfgfp::kan (Q339A, Y340A, D343A) & This study \\
\hline GL816 & GL504 A16-tnaA-sfgfp::kan (E346A, E347A) & This study \\
\hline GL817 & GL504 A17-tnaA-sfgfp::kan (C352A, Q353A, Q354A) & This study \\
\hline GL818 & GL504 A18-tnaA-sfgfp::kan (D363A, K366A) & This study \\
\hline GL819 & GL504 A19-tnaA-sfgfp::kan (H370A, D374A, Q375A) & This study \\
\hline GL820 & GL504 A20-tnaA-sfgfp::kan (E384A, K387A, R392A) & This study \\
\hline GL821 & GL504 A21-tnaA-sfgfp::kan (S398A, R403A, D404A) & This study \\
\hline GL822 & GL504 A22-tnaA-sfgfp::kan (K406A, K409A, Q410A) & This study \\
\hline GL823 & GL504 A23-tnaA-sfgfp::kan (E416A, R419A) & This study \\
\hline GL824 & GL504 A24-tnaA-sfgfp::kan (T426A, Y427A, T428A) & This study \\
\hline GL825 & GL504 A25-tnaA-sfgfp::kan (Q429A, T430A, H431A, D433A) & This study \\
\hline GL826 & GL504 A26-tnaA-sfgfp::kan (E437A, K440A, H441A) & This study \\
\hline GL827 & GL504 A27-tnaA-sfgfp::kan (K443A, E444A, N445A, N448A) & This study \\
\hline GL828 & GL504 A28-tnaA-sfgfp::kan (K450A) & This study \\
\hline
\end{tabular}


Table 5 E. coli strains (Continued)

\begin{tabular}{|c|c|c|}
\hline GL829 & GL504 A29-tnaA-sfgfp::kan (T453A, T455A, Y456A, E457A) & This study \\
\hline GL830 & GL504 A30-tnaA-sfgfp::kan (K459A) & This study \\
\hline GL831 & GL504 A31-tnaA-sfgfp::kan (R462A, H463A, T465A) & This study \\
\hline GL832 & MG1655 A32-tnaA-sfgfp::kan (K467A, K469A, E470A) & This study \\
\hline GL833 & GL504 A21a-tnaA-sfgfp::kan (S398A) & This study \\
\hline GL834 & GL504 A21b-tnaA-sfgfp::kan (R403A) & This study \\
\hline GL835 & GL504 A21c-tnaA-sfgfp::kan (D404A) & This study \\
\hline GL836 & GL504 A23a-tnaA-sfgfp::kan (E416A) & This study \\
\hline GL837 & GL504 A23b-tnaA-sfgfp::kan (R419A) & This study \\
\hline GL838 & GL504 A31a-tnaA-sfgfp::kan (R462A) & This study \\
\hline GL839 & GL504 A31b-tnaA-sfgfp::kan (H463A) & This study \\
\hline GL840 & GL504 A31c-tnaA-sfgfp::kan (T465A) & This study \\
\hline GL844 & GL504 A33-tnaA-sfgfp::kan (K5A, K115A, K156A, K239A, K450A, K459A) & This study \\
\hline GL802D & GL504 A2-D1D3-sfgfp::kan (E9A, R12A, R14A) & This study \\
\hline GL803D & GL504 A3-D1D3-sfgfp::kan (E17A, K20A, R21A) & This study \\
\hline GL804D & GL504 A4-D1D3-sfgfp::kan (T23A, R24A, Y26A) & This study \\
\hline GL805D & GL504 A5-D1D3-sfgfp::kan (R27A, E28A, E29A) & This study \\
\hline GL806D & GL504 A6-D1D3-sfgfp::kan (K33A, S34A) & This study \\
\hline GL807D & GL504 A7-D1D3-sfgfp::kan (D42A, S43A, E44A, D45A) & This study \\
\hline GL808D & GL504 A8-D1D3-sfgfp::kan (D49A, T52A, D53A, S54A) & This study \\
\hline GL809D & GL504 A9-D1D3-sfgfp::kan (T60A, Q61A, S62A, Q64A) & This study \\
\hline GL814D & GL504 A14-D1D3-sfgfp::kan (N327A, D329A) & This study \\
\hline GL815D & GL504 A15-D1D3-sfgfp::kan (Q339A, Y340A, D343A) & This study \\
\hline GL816D & GL504 A16-D1D3-sfgfp::kan (E346A, E347A) & This study \\
\hline GL817D & GL504 A17-D1D3-sfgfp::kan (C352A, Q353A, Q354A) & This study \\
\hline GL818D & GL504 A18-D1D3-sfgfp::kan (D363A, K366A) & This study \\
\hline GL819D & GL504 A19-D1D3-sfgfp::kan (H370A, D374A, Q375A) & This study \\
\hline GL820D & GL504 A20-D1D3-sfgfp::kan (E384A, K387A, R392A) & This study \\
\hline GL821D & GL504 A21-D1D3-sfgfp::kan (S398A, R403A, D404A) & This study \\
\hline GL822D & GL504 A22-D1D3-sfgfp::kan (K406A, K409A, Q410A) & This study \\
\hline GL823D & GL504 A23-D1D3-sfgfp::kan (E416A, R419A) & This study \\
\hline GL824D & GL504 A24-D1D3-sfgfp::kan (T426A, Y427A, T428A) & This study \\
\hline GL825D & GL504 A25-D1D3-sfgfp::kan (Q429A, T430A, H431A, D433A) & This study \\
\hline GL826D & GL504 A26-D1D3-sfgfp::kan (E437A, K440A, H441A) & This study \\
\hline GL827D & GL504 A27-D1D3-sfgfp::kan (K443A, E444A, N445A, N448A) & This study \\
\hline GL829D & GL504 A29-D1D3-sfgfp::kan (T453A, T455A, Y456A, E457A) & This study \\
\hline GL831D & GL504 A31-D1D3-sfgfp::kan (R462A, H463A, T465A) & This study \\
\hline GL832D & GL504 A32-D1D3-sfgfp::kan (K467A, K469A, E470A) & This study \\
\hline GL833D & GL504 A21a-D1D3-sfgfp::kan (S398A) & This study \\
\hline
\end{tabular}

${ }^{a}$ frt indicates the presence of the following oligonucleotide scar that was left after removing the kan cassette: GAAGTCCTATACTITCTAGAGAATAGGAACTTC. The "A\#\#" numbers correspond to the site-directed mutations described in Tables 1 and 2.

that the sequestration of TnaA in discrete foci may regulate its enzyme activity. In addition, altering any one of three residues at the base of a loop near the catalytic pocket produces aberrant TnaA foci that exhibit greatly decreased tryptophanase activity. These latter results strongly imply that the disposition of this loop controls the formation of TnaA foci and may regulate the rate at which tryptophan can access the active site. 


\section{Methods}

Strains, plasmids and growth conditions

All strains were derivatives of E. coli MG1655, and are listed in Table 5. Strain GL60 was the source for the $i b p A$ $m$ Cherry::kan allele [25]. The plasmid pTnaA $\left(\operatorname{Kan}^{\mathrm{R}}\right)$ carries the wild type tnaA gene under control of the lac promoter [25]. The plasmid pJDT1 $\left(\mathrm{Amp}^{\mathrm{R}}\right)$ contains torAgfpmut 3 and was used as the template to amplify gfpmut3 [47]. Strains were selected by adding kanamycin (Kan, $50 \mu \mathrm{g} / \mathrm{ml}$ ) or ampicillin (Amp, $100 \mu \mathrm{g} / \mathrm{ml}$ ). Cells were grown overnight in LB medium supplemented with $0.4 \%$ glucose. Overnight cultures were diluted 1:100 into $2 \mathrm{ml}$ LB or M9 minimal medium supplemented with $2 \mathrm{mM}$ $\mathrm{MgSO}_{4}, 0.1 \mathrm{mM} \mathrm{CaCl}$, $33 \mu \mathrm{M}$ thiamine, $1 \%$ Bacto Casamino acids and $0.5 \mathrm{mM}$ tryptophan. Cells were incubated with shaking at $37^{\circ} \mathrm{C}$ for TnaA localization and indole production experiments. When applicable, $50 \mu \mathrm{M}$ IPTG was added to induce expression of the plasmidencoded tnaA gene.

\section{Strain construction}

Chromosomal gene deletions or insertions were performed by using $\lambda$-Red recombination [48]. The DNA fragments were amplified by one-step or sequential PCR using designated primers (see Additional file 1). To construct the D1-sfGFP and D1D2-sfGFP strains, the sfgfp:: $k a n$ cassettes were amplified from the chromosome of $E$. coli GL38 (aer-sfgfp::kan) and inserted into the chromosome of MG1655 to delete the appropriate subdomain fragments and to fuse sfgfp to $D 1$ and D1D2. To construct the D2-sfGFP, D3-sfGFP, D2D3-sfGFP and D1D3sfGFP strains, the respective $s f g f p$-containing fragments were amplified from the chromosome of GL680 (D1D2sfgfp::kan) or GL40 (tnaA-sfgfp::kan) and inserted into the chromosome of GL504 ( $\Delta t n a A:: f r t)$ under control of the original tha promoter. The A1-TnaA-sfGFP to A31TnaA-sfGFP strains were constructed by amplifying the tnaA-sfgfp::kan fragments from the chromosome of GL40 and inserting them into the chromosome of GL504 at the original tnaA gene location. To construct the A32-TnaA-sfGFP strain, the sfgfp::kan fragment was amplified from the chromosome of E. coli GL38 and inserted into the chromosome of MG1655 to introduce the mutation and to fuse $s f g f p$ to tnaA. To construct the A33-TnaA-sfGFP strain, the DNA fragment was generated by six rounds of PCR reactions to introduce the mutations for the six codons one by one. The A1-D1D3sfGFP to A32-D1D3-sfGFP fusions were constructed in the same way as described for the D1D3-sfGFP fusions, except that the chromosomes of corresponding A1TnaA-sfGFP to A32-TnaA-sfGFP strains were used as the templates for PCR. To construct the TnaAmGFPmut3 fusion, the fragment encoding mGFPmut3 was amplified from gfpmut3 in pJDT1 by using two- step PCR to introduce the A206K mutation [29]. The mgfpmut3 fragment was joined to the kan cassette by an additional PCR and then inserted into the chromosome of MG1655 to replace the stop codon of tnaA. The D1D3-mGFPmut3 fusion was constructed as described for the D1D3-sfGFP fusion, except that the relevant gene fragment was amplified from the chromosome of GL676 (tnaA-mgfpmut3::kan). The entire tnaA region in all strains was PCR amplified and sequenced to confirm the presence of the correct gene constructions.

\section{TnaA structure modeling}

The crystal structures of TnaA (open conformation, PDB 2OQX; closed conformation, PDB 2C44) were assembled into tetramers by using the PISA software (http://www.ebi.ac.uk/pdbe/prot_int/pistart.html) [49]. In silico analysis and visualization of TnaA structures were performed by using the PyMOL Molecular Graphics System, Version 1.3. Schrödinger, LLC (http://www. pymol.org).

\section{Microscopy}

Phase contrast and fluorescence images of cells were obtained by using an Olympus microscope BX60 with a $100 \times$ oil objective (1.3 NA PH3), a 1.4 MP MONO CCD camera XM10, and illuminated by the fluorescence source X-Cite120. Live cells were loaded to $1 \%$ agarosecoated slides for imaging. GFP was visualized by using the OSF-0008Z filter set (471 nm excitation/520 nm emission).

\section{TnaA enzyme activity and protein stability}

TnaA activity was assayed by measuring indole production [19]. Standard curves with known concentrations of indole confirmed that the assay was linear for indole amounts down to $0.01 \mathrm{mM}$. Samples were collected at different points during cell growth and the amounts of indole were determined as described previously [19]. Cellular proteins were separated by SDS-PAGE, and TnaA and its various protein fragments that had been fused to sfGFP were visualized by in-gel GFP fluorescence imaging [19].

\section{Additional file}

Additional file 1: Table S1. Title of dataset: Oligonucleotides used to construct thaA mutants. Description of dataset: list of oligonucleotides used in making mutant genes.

\section{Competing interests}

The authors declare that they have no competing interests.

\section{Authors' contributions}

GL and KDY conceived the project, devised the experiments, analyzed the data, interpreted the results and wrote the paper. GL performed the experiments. Both authors read and approved the final manuscript. 


\section{Acknowledgements}

Funding for this project was provided by the US Government and managed by the Army Research Office under Award No. W911NF-10-1-0058. All clones were sequenced by the UAMS Sequencing Core, which is supported by $\mathrm{NIH}$ COBRE grant P20 GM103625, Center for Microbial Pathogenesis and Host Inflammatory Responses.

\section{Received: 23 July 2014 Accepted: 13 January 2015 Published online: 04 February 2015}

\section{References}

1. Newton WA, Morino Y, Snell EE. Properties of crystalline tryptophanase. J Biol Chem. 1965;240:1211-8.

2. Lee $\mathrm{JH}$, Lee J. Indole as an intercellular signal in microbial communities. Fems Microbiol Rev. 2010;34:426-44.

3. Kamaraju K, Smith J, Wang JX, Roy V, Sintim HO, Bentley WE, et al. Effects on membrane lateral pressure suggest permeation mechanisms for bacterial quorum signaling molecules. Biochemistry. 2011;50:6983-93.

4. Pinero-Fernandez S, Chimerel C, Keyser UF, Summers DK. Indole transport across Escherichia coli membranes. J Bacteriol. 2011;193:1793-8.

5. Wikoff WR, Anfora AT, Liu J, Schultz PG, Lesley SA, Peters EC, et al. Metabolomics analysis reveals large effects of gut microflora on mammalian blood metabolites. Proc Natl Acad Sci U S A. 2009;106:3698-703.

6. Han TH, Lee JH, Cho MH, Wood TK, Lee J. Environmental factors affecting indole production in Escherichia coli. Res Microbiol. 2011;162:108-16.

7. Vega NM, Allison KR, Samuels AN, Klempner MS, Collins JJ. Salmonella typhimurium intercepts Escherichia coli signaling to enhance antibiotic tolerance. Proc Natl Acad Sci U S A. 2013;110:14420-5.

8. Molina-Santiago C, Daddaoua A, Fillet S, Duque E, Ramos JL. Interspecies signalling: Pseudomonas putida efflux pump TtgGHI is activated by indole to increase antibiotic resistance. Environ Microbiol. 2014;16:1267-81.

9. Kim J, Park W. Indole inhibits bacterial quorum sensing signal transmission by interfering with quorum sensing regulator folding. Microbiol. 2013;159:2616-25.

10. Shimada Y, Kinoshita M, Harada K, Mizutani M, Masahata K, Kayama H, et al Commensal bacteria-dependent indole production enhances epithelial barrier function in the colon. PLoS One. 2013;8:e80604.

11. Mercado J, Valenzano MC, Jeffers C, Sedlak J, Cugliari MK, Papanikolaou E, et al. Enhancement of tight junctional barrier function by micronutrients: compound-specific effects on permeability and claudin composition. PLoS One. 2013;8:e78775.

12. Stewart $\mathrm{V}$, Yanofsky $\mathrm{C}$. Evidence for transcription antitermination control of tryptophanase operon expression in Escherichia coli K-12. J Bacteriol. 1985;164:731-40.

13. Deeley MC, Yanofsky C. Nucleotide sequence of the structural gene for tryptophanase of Escherichia coli K-12. J Bacteriol. 1981;147:787-96.

14. Sarsero JP, Wookey PJ, Gollnick P, Yanofsky C, Pittard AJ. A new family of integral membrane proteins involved in transport of aromatic amino acids in Escherichia coli. J Bacteriol. 1991;173:3231-4.

15. Deeley MC, Yanofsky C. Transcription initiation at the tryptophanase promoter of Escherichia coli K-12. J Bacteriol. 1982;151:942-51.

16. Stewart V, Landick R, Yanofsky C. Rho-dependent transcription termination in the tryptophanase operon leader region of Escherichia coli K-12. J Bacteriol. 1986;166:217-23.

17. Cruz-Vera LR, Gong M, Yanofsky C. Changes produced by bound tryptophan in the ribosome peptidyl transferase center in response to TnaC, a nascent leader peptide. Proc Natl Acad Sci U S A. 2006;103:3598-603.

18. Yanofsky C, Horn V, Gollnick P. Physiological studies of tryptophan transport and tryptophanase operon induction in Escherichia coli. J Bacteriol. 1991;173:6009-17.

19. Li G, Young KD. Indole production by the tryptophanase TnaA in Escherichia coli is determined by the amount of exogenous tryptophan. Microbiol. 2013;159:402-10.

20. London J, Goldberg ME. The tryptophanase from Escherichia coli K-12. I. Purification, physical properties, and quaternary structure. J Biol Chem. 1972;247:1566-70.

21. Kagamiyama H, Matsubara $H$, Snell EE. The chemical structure of tryptophanase from Escherichia coli. 3. Isolation and amino acid sequence of the tryptic peptides. J Biol Chem. 1972;247:1576-86.
22. Erez T, Gdalevsky G, Torchinsky YM, Phillips RS, Parola AH. Cold inactivation and dissociation into dimers of Escherichia coli tryptophanase and its W330F mutant form. Biochim Biophys Acta. 1998;1384:365-72.

23. Ku SY, Yip P, Howell PL. Structure of Escherichia coli tryptophanase. Acta Crystallogr D Biol Crystallogr. 2006;62:814-23.

24. Tsesin N, Kogan A, Gdalevsky GY, Himanen JP, Cohen-Luria R, Parola AH, et al. The structure of apo tryptophanase from Escherichia coli reveals a wide-open conformation. Acta Crystallogr D Biol Crystallogr. 2007;63:969-74.

25. Li G, Young KD. Isolation and identification of new inner membraneassociated proteins that localize to cell poles in Escherichia coli. Mol Microbiol. 2012;84:276-95.

26. Li G, Young KD. A cAMP-independent carbohydrate-driven mechanism inhibits tnaA expression and TnaA enzymatic activity in Escherichia coli. Microbiol. 2014;160:2079-88.

27. Lindner AB, Madden R, Demarez A, Stewart EJ, Taddei F. Asymmetric segregation of protein aggregates is associated with cellular aging and rejuvenation. Proc Natl Acad Sci U S A. 2008;105:3076-81.

28. Van der Henst C, Charlier C, Deghelt M, Wouters J, Matroule JY, Letesson JJ, et al. Overproduced Brucella abortus PdhS-mCherry forms soluble aggregates in Escherichia coli, partially associating with mobile foci of IbpA-YFP. BMC Microbiol. 2010;10:248.

29. Landgraf D, Okumus B, Chien P, Baker TA, Paulsson J. Segregation of molecules at cell division reveals native protein localization. Nat Methods. 2012;9:480-2.

30. Zhang J, Sprung R, Pei J, Tan X, Kim S, Zhu H, et al. Lysine acetylation is a highly abundant and evolutionarily conserved modification in Escherichia coli. Mol Cell Proteomics. 2009:8:215-25.

31. Yu BJ, Kim JA, Moon JH, Ryu SE, Pan JG. The diversity of lysine-acetylated proteins in Escherichia coli. J Microbiol Biotechnol. 2008;18:1529-36.

32. Kogan A, Gdalevsky GY, Cohen-Luria R, Goldgur Y, Phillips RS, Parola AH, et al. Conformational changes and loose packing promote $E$. coli tryptophanase cold lability. BMC Struct Biol. 2009;9:65.

33. Rudner DZ, Losick R. Protein subcellular localization in bacteria. Cold Spring Harb Perspect Biol. 2010;2:a000307.

34. Dworkin J. Cellular polarity in prokaryotic organisms. Cold Spring Harb Perspect Biol. 2009;1:a003368.

35. de Boer PA. Advances in understanding E. coli cell fission. Curr Opin Microbiol. 2010;13:730-7.

36. Erhardt M, Namba K, Hughes KT. Bacterial nanomachines: the flagellum and type III injectisome. Cold Spring Harb Perspect Biol. 2010;2:a000299.

37. Scott ME, Dossani ZY, Sandkvist M. Directed polar secretion of protease from single cells of Vibrio cholerae via the type II secretion pathway. Proc Natl Acad Sci U S A. 2001;98:13978-83.

38. Kumar RB, Das A. Polar location and functional domains of the Agrobacterium tumefaciens DNA transfer protein VirD4. Mol Microbiol. 2002:43:1523-32

39. Vignon G, Kohler R, Larquet E, Giroux S, Prevost MC, Roux P, et al. Type IVlike pili formed by the type II secreton: specificity, composition, bundling, polar localization, and surface presentation of peptides. J Bacteriol. 2003;185:3416-28.

40. Wu LJ, Errington J. RacA and the Soj-Spo0J system combine to effect polar chromosome segregation in sporulating Bacillus subtilis. Mol Microbiol. 2003;49:1463-75

41. Judd PK, Kumar RB, Das A. Spatial location and requirements for the assembly of the Agrobacterium tumefaciens type IV secretion apparatus. Proc Natl Acad Sci U S A. 2005;102:11498-503.

42. Toro E, Shapiro L. Bacterial chromosome organization and segregation. Cold Spring Harb Perspect Biol. 2010;2:a000349.

43. Michaelis AM, Gitai Z. Dynamic polar sequestration of excess MurG may regulate enzymatic function. J Bacteriol. 2010;192:4597-605.

44. Rothe FM, Wrede C, Lehnik-Habrink M, Görke B, Stülke J. Dynamic localization of a transcription factor in Bacillus subtilis: the LicT antiterminator relocalizes in response to inducer availability. J Bacteriol. 2013;195:2146-54

45. Ingerson-Mahar M, Briegel A, Werner JN, Jensen GJ, Gitai Z. The metabolic enzyme CTP synthase forms cytoskeletal filaments. Nat Cell Biol. 2010;12:739-46

46. Swulius MT, Jensen GJ. The helical MreB cytoskeleton in Escherichia coli MC1000/pLE7 is an artifact of the N-Terminal yellow fluorescent protein tag. J Bacteriol. 2012;194:6382-6. 
47. Thomas JD, Daniel RA, Errington J, Robinson C. Export of active green fluorescent protein to the periplasm by the twin-arginine translocase (Tat) pathway in Escherichia coli. Mol Microbiol. 2001;39:47-53.

48. Datsenko KA, Wanner BL. One-step inactivation of chromosomal genes in Escherichia coli K-12 using PCR products. Proc Natl Acad Sci U S A. 2000;97:6640-5.

49. Krissinel E, Henrick K. Inference of macromolecular assemblies from crystalline state. J Mol Biol. 2007;372:774-97.

Submit your next manuscript to BioMed Central and take full advantage of:

- Convenient online submission

- Thorough peer review

- No space constraints or color figure charges

- Immediate publication on acceptance

- Inclusion in PubMed, CAS, Scopus and Google Scholar

- Research which is freely available for redistribution 